\title{
Off-Specular Reflectivity Study of Sputter-Deposition of Platinum during Growth
}

\author{
H. You, K.G. Huang, and R.T. Kampwirth \\ Materlals Science Division, Argonne National Laboratory, Argonne, IL 60439 \\ USA
}

Materials Science Division

Argonne National Laboratory

Argonne, Ill 60439

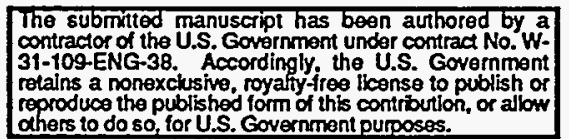

August 1995

jjc

DISCLAIMER

Distribution:

1-2. M. J. Masek

3. B. D. Dunlap

4. P. A. Montano

5. F. Y. Fradin

6. R. Gottschall

7. Editorial Office

8. Authors

Submitted to Physica B, the proceedings of the Fourth International Conference on Surface X-Ray and Neutron Scattering, Lake Geneva, WI, USA, June 25-30, 1995.

This work is supported by the Division of Materials Sciences, Office of Basic Energy Sciences of DOE, under contract No. W-31-109-ENG-38. 


\title{
Off-Specular Reflectivity Study of Sputter-Deposition of Platinum during Growth
}

\author{
H. You, K.G. Huang, and R.T. Kampwirth \\ Materials Science Division, Argonne National Laboratory, Argonne, IL 60439 \\ $U S A$
}

(Received; Aug. 21, 1995)

\begin{abstract}
A series of off-specular diffuse scattering measurements were performed by scanning the detector arm parellel to the sample surface during growth of platinum films on polished silicon substrates. During early stage of growth; we observe a halo of diffuse scattering around the specular reflection which is similar to Henzler's ring. [P. Hahn, J. Clabes; and M. Henzler, J. Appl. Phys. 51,2079 (1980)] A model of random islands was developed to explain the halo of diffuse scattering. During late growth, the film surface was consistent with a partially self-affine surface.
\end{abstract}

\section{INTRODU்CTION}

Deposition and growth on flat substrates is one of most common modern materials processing methods. During deposition, it is desirable to monitor the growth in situ using structural probes such as electrons and x-rays. Commonly electron-based techniques were chosen because it is relatively easier to produce and control electrons than $\mathrm{x}$-rays. However, in non-ultra-high-vacuum materials processing such as sputter-deposition and liquid phase epitaxy, electrons can not be used and x-rays become virtually only possible probe to monitor the growth. 
Among several $x$-ray techniques to monitor the growth, x-ray reflectivity measurements have been gaining the popularity in recent years. Recently; t:e have used specular x-ray reflectivity measurements to study a dynamic scaling behavior during.sputter-growth of gold films on polished silicon substrates. [1] While the specular $x$-ray reflectivity is powerful in measuring surface-normal electron density profile, off-specular x-ray diffuse scattering provides a considerably more detailed information on the in-plane nature of the surface. -More recently we performed a series of specular and off-specular reflectivity measurements during growth of platinum films on polished silicon in a similar way to the previous study. [1] In this proceeding, we will focus only on the off-specular measurements and developing. models to fit them.

In the early stage of film growth, we find. a halo of scattering around the specular reflection. signifying the growth of microscopic platinum islands. As the deposition continues, islands . gradually fill the surface and their sizes continuously grow to form a homogeneously rough surface. For the initial stage of growth, the off-specular reflectivity will be modeled by. scattering from a system of random islands. For the late stage of growth, it will be modeled by that from a partially self-affine surface.

\section{EXPERIMENTAL}

. The $\mathrm{x}$-ray reflectivity measurements were performed in situ using a high vacuum chamber equipped with a faced-magnetron sputtering system and x-ray transparent windows. A schematic diagram of the chamber is given in Fig.1. This chamber was fabricated by. modifying the chamber previously used. [2] Axial magnetic and radial electric fields ionize . Ar gas and accelerate the positive Ar ions towards the targets with sufficient energy to remove (sputter) neutral platinum atoms. The sputtered platinum atoms form a homogeneous "cloud" and deposit onto the substrate. The substrate is located at right angles to. the two faced targets and positioned to remain well below the edge of the plasma. The Ar gas pressure was held at 10 mTorr during deposition as measured by a convectron pressure 
gauge. The gun was operated at $600 \mathrm{~V}$ for deposition. The rate of deposition at $600 \mathrm{~V}$ mas $0.3 \hat{A} / \mathrm{sec}$ as determined from specular x-ray reflectivity measurements. While x-ray cata were being recorded, the power supplied to the faced-magnetron gun was reduced to $200 \mathrm{~V}$. At this potential, the amount of deposition during data acquisition was negligible but. at the same time, the plasma was kept active throughout the measurement.

The polished silicon substrates $(1.3 \mathrm{~cm}$ by $1.3 \mathrm{~cm}$ by $0.15 \mathrm{~cm}$ ) were ultrasonically çieaned for five minutes each in research grade acetone, ethanol, and distilled water. The substrates were blown dry using nitrogen gas and mounted onto a heating stage using highly conductive silver cement. Resistive heating was used to heat the sample to $600 \mathrm{~K}$ to flash clean the surface and kept at $400 \mathrm{~K}$ for deposition of platinum and $\mathrm{x}$-ray scans.

. The .x-ray measurements were performed at the National Synchrotron Light Source on beamline X22B. The incident. beam was focused in the horizontal and vertical directions by, a toroidal nickel-coated aluminum mirror. The incident beam spot size was $\sim 2 \mathrm{~mm}$. horizontal by $\sim 1 \mathrm{~mm}$ vertical. A single flat Ge(111) crystal monochromator was used and the incident x-ray wavelength chosen for the measurements was $1.74 \AA$. The measurements rere performed in the horizontal scattering geometry and the in-plane instrumental resolution was approximately $4 \times 10^{-3} \AA^{-1}$. The chamber was mounted onto the phi circle of a four-circle diffractometer, and scintillation detectors were used to monitor the incident and reflected x-ray. beam intensities. The detector path and slit assembly was tilted up by $2.5^{\circ}$ with respect to the incoming beam and the sample by $1.25^{\circ}$ to achieve $0.15 \AA^{-1}$ surface normal momentum component at the nominal two theta zero. Then for the off-specular components, the two theta was scanned to $\pm 8^{\circ}$ at which the beam was blocked by the window of the sputtering chamber. 


\section{KINEMATIC SCATTERING EQUATIONS OF.HARD CORE SYSTEMS}

\section{A. A system of identical hard-core objects}

In this section we will consider scattering equations from 2-d random hard core systems. First, we will consider a simplest system of hard core objects whose positional distribution is completely random. An example of such random hard core systems in three dimension is a system of gas. A scattering equation for 3-d gas systems was first obtained by Debye [3]. The beginning of this section will therefore follow the Debye's derivation to obtain a . two-dimensional analogue.

We will begin by calculating $x$-ray scattering intensity from a random distribution of $N$ identical hard core disks with a radius $a$ and zero thickness on a surface with area $S$. Based on the First Born approximation, the scattering intensity $I(Q)$ can be written into two terms; summation of the form factor and summation of the cross terms.

$$
\begin{aligned}
I(\mathrm{Q}) & =\sum_{p}\left|F_{p}\right|^{2}+\sum_{p \neq q} \sum F_{p} F_{q} e^{i \mathrm{Q} \cdot\left(\mathrm{x}_{p}-\mathrm{x}_{q}\right)} \\
\therefore \quad & =|F|^{2}\left(N+\frac{N(N-1)}{S^{2}} \iint W\left(\mathrm{x}_{p, q}\right) e^{i \mathrm{Q} \cdot\left(\mathrm{x}_{p}-\mathrm{x}_{q}\right)} d \mathrm{x}_{p} d \mathrm{x}_{q}\right)
\end{aligned}
$$

where $W\left(\mathrm{x}_{p, q}\right)$ is the probability that the center of disk $p$ lies in the element of surface $d s_{p}$ and that of disk $q$ lies in the element of surface $d s_{q}$. In evaluating the double integral in Eq.(2) let us assume that the surface is translationally and orientationally isotropic therefore the function $W\left(\mathrm{x}_{p, \dot{q}}\right)$ is azimuthally symmetric. In this case, the function $W\left(\mathrm{x}_{p, q}\right)$ is a step

. function in $x$ which is equal to zero for $x<.2 a$ since the nearest distance between two neighbors is $2 a$. And it is unity for $x>2 a$ since they occupy the rest of the surface area $4 S-4 \pi a^{2}$ with an equal probability. (The surface area is $4 S$ since $\mathrm{x}_{p}-\mathrm{x}_{q}$ spans twice as much as $\mathrm{x}_{p}$ or $\mathrm{x}_{q}$ alone.) The function $W(x)$ for this case is schematically shown in Fig. 2(a). With a coordinate transformation the double surface elements $d s_{p} d s_{q}$ becomes $d^{2} \mathrm{x} d^{2} \mathrm{X}$ where $\mathrm{X}=\left(\mathrm{x}_{p}+\mathrm{x}_{q}\right) / 2$ and $\mathrm{x}=\mathrm{x}_{p}-\mathrm{x}_{q}$ and Eq.(2) becomes independent of $X$,

$$
I(\mathrm{Q})=|F|^{2}\left(N-\frac{N(N-1)}{4 S} \int\left[1-W\left(\mathrm{x}_{p, q}\right)\right] e^{i \mathrm{Q} \cdot \mathbf{x}} d \mathbf{x}\right)+|F|^{2} N(N-1) \frac{2 J_{1}(Q D)}{Q D}
$$


where $D$ is the x-ray coherence length. In the intergand of the first term, $1-W(x)$ was used instead of $W(x)$ by adding the specular term at the end. Then the integral in the first term is essentially the scattering amplitude from a disk with a radius $2 a$ and the corresponding equation for the off-specular scattering can be simplified to

$$
\begin{aligned}
I(\mathrm{Q}) & =N|F(Q a)|^{2}\left(1-\frac{(N \cdot 1)}{4 S} F(2 Q a)\right) \\
& =\pi a^{2} \Lambda S\left(\frac{2 J_{1}(Q a)}{Q a}\right)^{2}\left(1-\Lambda \frac{J_{1}(2 Q a)}{Q a}\right)
\end{aligned}
$$

where $\Lambda=4 \pi a^{2}(N-1) / 4 S$ is the coverage of the disks.

Now let us consider the distribution function $W(x)$ shown in Fig. 2(b). It has higher probability distribution for $2 a<x \leq n a$ than the rest of the surface area by an excess . probability of $\Lambda^{\prime}$. Since the function shown in (b) is equivalent to the difference between (c) and (d), the scattering intensity can easily be written as,

$$
I(\mathrm{Q})=N|F|^{2}\left[1-\left(\Lambda+\Lambda^{\prime}\right) \frac{J_{1}\left(2 Q_{11} a\right)}{Q_{11} a}+\left(\Lambda+\Lambda^{\prime}\right) \frac{2 J_{1}\left(n Q_{11} a\right)}{n Q_{11} a}\right]
$$

In this equation, we can substitute $n$ by $2 \sqrt{1+\Lambda / \Lambda^{\prime}}$ if we impose a constraint, $2 \pi \int_{0}^{\infty}[1:-$ $W(x)] x d x=0$. This constraint means simply that the probability excluded due to the hard-core repulsion is all added to the $x$ range of $2 a<x \leq n a$.

We can now rewrite this equation with a three dimensional vector $Q$ for more generally shaped hard-core objects distributed over a surface. Since the positional distribution of the hard-core system is still two-dimensional, the scattering intensity per unit surface area can be rewritten as,

$$
I(\mathrm{Q})=N|F(\mathrm{Q})|^{2}\left[1-\left(\Lambda+\Lambda^{\prime}\right) F_{11}\left(2 \mathrm{Q}_{11} \cdot \mathrm{x}\right) \div\left(\Lambda+\Lambda^{\prime}\right) F_{11}\left(n \mathrm{Q}_{11} \cdot \mathrm{x}\right)\right]
$$

where $F(\mathrm{Q})$ is the three-dimensional form factor of a single object and $F_{11}$ are twodimensional form factor due to the hard-core exclusion which depends only on the foot print of the hard-core object. When the orientation and the size of the object are identical but randomly distributed over the surface, $f_{11}$ is a two dimensional Fourier transformation of 
twice the size of the foot print of the object. For an example, a system of spheres randomly located on surface will have a scattering intensity of

$$
I(\mathrm{Q})=N\left|4 \pi a^{3}\left(\frac{\sin Q a}{(Q a)^{3}}-\frac{\cos Q a}{(Q a)^{2}}\right)\right|^{2}\left[1-\left(\Lambda+\Lambda^{\prime}\right) \frac{J_{1}\left(2 Q_{11} a\right)}{Q_{11} a}+\left(\Lambda+\Lambda^{\prime}\right) \frac{2 J_{1}\left(n Q_{\mathrm{n}} a\right)}{n Q_{11} a}\right] .
$$

where $a$ is a radius of the sphere and $\Lambda$ is the fractional coverage of the sphere each of which occupies $\pi a^{2}$. For a system of hollow spheres the form factor $(|\cdots|)$ should be replaced by $\left|4 \pi a^{2} \frac{\sin Q a}{Q a}\right|$.

Following the similar vein, we can obtain an equation for a system of rectangular islands whose dimensions are $a \times b$ as,

$I(\mathrm{Q})=a b \Lambda\left|\frac{\sin \left(Q_{x} a / 2\right)}{Q_{x} a / 2} \frac{\sin \left(Q_{y} b / 2\right)}{Q_{y} b / 2}\right|^{2} \cdot\left[1-\left(\Lambda+\Lambda^{\prime}\right) \frac{\sin \left(Q_{x} a\right)}{Q_{x} a} \frac{\sin \left(Q_{y} b\right)}{Q_{y} b}+\left(\Lambda+\Lambda^{\prime}\right) \frac{\sin \left(n Q_{x} a\right)}{n Q_{x} a} \frac{\sin \left(n Q_{y} b\right)}{n Q_{y} b}\right]$.

where $n=\sqrt{1+\Lambda / \Lambda^{\prime}}$. We assume here that the shape of distribution function $W(\mathrm{x})$. also maintain the aspect ratio of the islands. Similarly one can find the scattering intensity from: . a system of hard core objects with a different shape or with different pile-up probability.

\section{.. B. Hard Core Objects with a Statistical Distribution in Size}

When the objects are not identical but has a statistical distribution in size and in shape, Eq. (2) should be modified to reflect the statistical distribution. In general, the statistical averaging should be performed case by case to reflect the details of the system such as the shapes of average objects, the distribution of the sizes, and the distribution of orientations. However, in this section, we will examine a system of disks to seek general features of the scattering from a more complicated system such as an assembly of microscopic islands grown on the surface.

Since the intensity that we measure is an incoherent sum of many photon events, we can calculate the scattering intensity via an ensemble average. In particular, for a system where their positions are chosen independently from their sizes, the averaging can be performed separately over the two terms in Eq. (1). 


$$
I(\mathrm{Q})=N\left\langle|F(\mathrm{Q})|^{2}\right\rangle_{A V^{\prime} E}+\frac{N(N-1)}{S}\left|\langle F(\mathrm{Q})\rangle_{A V E}\right|^{2}\left\langle e^{i \mathrm{Q} \cdot\left(\mathrm{x}_{p}-\mathrm{x}_{\mathrm{q}}\right)}\right\rangle_{A I^{\prime} E}
$$

Here, of course, their positions are not independent variables but dependent on the relative distance $\mathrm{x}_{p}-\mathrm{x}_{q}$. For example, the closest possible distance between a pair of disks is limi $=\mathrm{d}$ to the sum of their radii and statistically averaged pair distribution probability should be ize same as the robability distribution of disk size except that the distribution width is twice as large. Notice that in the first term of this equation the average is performed on the absolvie square of the form factor while in the second term the average is performed on the form factor itself then squared. In general these two average would not yield an identical function. However, we assume here for analytical simplicity that $\left\langle|F(Q)|^{2}\right\rangle_{A V E} \approx\left|\langle F(Q)\rangle_{A V E}\right|^{2}$. T:is assumption is asymptotically valid for large $Q$ and it can be shown easily for the form facturs discussed in the previous section. Now it can be rewritten in a form similar to Eq: (5) as

$$
I(\mathbf{Q})=N|\langle F(\mathbf{Q})\rangle|^{2}\left[1-\left(\Lambda+\Lambda^{\prime}\right)\left\langle\frac{J_{1}\left(2 Q_{11} a\right)}{Q_{11} a}\right\rangle+\left(\Lambda \cdot+\Lambda^{\prime}\right)\left\langle\frac{J_{1}\left(2 \sqrt{1+\Lambda / \Lambda^{\prime}} Q_{n} a\right)}{\sqrt{1+\Lambda / \Lambda^{\prime}} Q a}\right\rangle\right]
$$

Since the oscillatory behavior of function. $J_{1}\left(2 Q_{11} a\right) / Q_{11} a$ and the form factor will in general be damped via the average over the size distribution. Let us now assume a following distribution of the disk sizes,

$$
P(a)=\frac{1}{2 a_{0}^{3}} a^{2} e^{-a / a_{0}}
$$

where the mean radius is $3 a_{0}$ and the variance is $3 a_{0}^{2}$. Advantage of this distribution function is in mathematical simplicity. This is one of a few cases where one can find a closed form in the integral of $\int P(a) 2 J_{1}\left(Q_{11} a\right) / Q_{11} a d a$ and the result of the integral is $1 /\left[1+\left(Q_{11} a_{0}\right)^{2}\right]^{3}$. Finally the intensity becomes

$$
I\left(Q_{11}\right)=\frac{\Lambda}{\left[1+\left(Q_{11} a_{0}\right)^{2}\right]^{2+2 \chi}}\left[1-\frac{\Lambda+\Lambda^{\prime}}{\left[1+\left(2 Q_{11} a_{0}\right)^{2}\right]^{3 / 2}}+\frac{\Lambda+\Lambda^{\prime}}{\left[1+\left(1+\Lambda / \Lambda^{\prime}\right)\left(2 Q_{11} a_{0}\right)^{2}\right]^{3 / 2}}\right]
$$

where $\chi=0.5$. When the object is not an ideal disk, the form factor average $\left\{F_{p}\right\rangle$ should be modified accordingly. As an approximation let us write $\left\langle F_{p}\right\rangle \approx 1 /\left[1+\left(Q a_{0}\right)^{2}\right]^{1+\chi}$ as we have shown in the above equation. This form of average form factor can be justifi:d 
from various examples where no analytically closed form can be found. For instance, if the shape of the islands is hemispherical, $\chi=2$ for large $Q$. In general we can define $\chi$ as a. fit parameter which signifies the aspect ratio of the hard core objects along with other fit. parameters such as $a_{0}, \Lambda, \Lambda^{\prime}$, and overall scale factor in our data analyses.

To show the general behavior of Eq. (8), $I(Q)$ is plotted for $a_{0}=1$ and $\chi=0.5$ and several possible values of $\Lambda$ and $\Lambda^{\prime}$ in Fig. 3. Wimen $\Lambda^{\prime}$ goes to zero, the third term in [ $\left.\cdots\right]$ of Eq. (8) will become a narrow function centered at $Q_{\mathrm{n}}=0$ and a part of specular scattering. Then the second term is proportional to $1-\Lambda$. For a close packed system $(\Lambda \lesssim 1)$, therefore, . the scattering intensity near the specular reflection will be small. An example for $\Lambda=0.9$ is shown as dashed line. As we can see the scattering intensity shows a very pronounce asymmetric prafile in the peak shape, sharply rising from $1-\Lambda$ but slowly falling in the . function of $Q_{11}$ following the square of the form factor. On the other hand when $\Lambda^{\prime}$ is finite, .

a peak, centered at $Q_{11}$ with a finite width inversely proportional to $a \sqrt{1+\Lambda / \Lambda^{\prime}}$, exists. Therefore, the intensity will fall for small $Q_{11}$ as dominated by the third term in the bracket. But it will increases for the intermediate $Q_{11}$ values as the second term dominates. Eventually the second term's contribution becomes also negligible and the intensity falls off with the square of the form factor. Such an example is shown with a solid line in the figure. The essential feature of Eq. (8) is similar to that of Eq. (5) except that the oscillatory behaviors are completely damped by. the averaging with the distribution function [Eq. (7)].

\section{RESULTS AND DISCUSSIONS}

A series of transverse scans are shown in Fig. 4. The $\mathrm{x}$-axis is for in-plane momentum

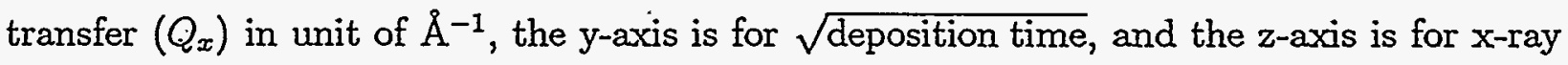
intensity shown in a log scale. The out-of-plane momentum transfer $\left(Q_{z}\right)$ was fixed at 0.15 $\AA^{-1}$ for the entire scans. Thus, the peak at $Q_{x}=0$ is the specular intensity at $Q_{z}=0.15 \AA^{-1}$. The first transverse scan shown here is made after deposition of platinum to approximately $35 \AA$ thick on the average. After that, the scans were made.after subsequent additional 
amount of deposition, initially in steps of several $\AA$ but later in steps of $\sim 1000 \AA$ until the total film thickness reaches approximately $9000 \AA$.

By examining the individual scans, it was clear that the scans can be separated into two. parts; specular peak and off-specular diffuse scattering. The last scan of the series is shown in linear and semi-log scales in Fig. 5. In this figure, the shoulders around $0.1 \AA^{-1}$ make fits to a single peak not possible. The distinction of the central and broad components was possible for all the scans shown in Fig. 4. Therefore, the scans were fit with two components. One is a narrow Gaussian component whose intensity and width are the adjustable parameters and the other is Eq. (8). In Fig. 6, the peak intensity (open circles) and width (filled circles) of the Gaussian components are plotted against the film thickness in a log-log plot. The peak intensity decreases gradually as the film thickness increases with a couple of exceptional minima near $70 \AA$ and $500 \AA$. The intensity minima are due to the destructive . interference between the reflection from the film/substrate interface and that from the film surface. Since the measurements were not continuously made to detect all the minima, only two of the minima happen to coincide with the measured points and shows pronounce dips. However, the important characteristic on which we would like to focus here is the fact that intensity gradually decrease as the film thickmess increases. This characteristic can be understood largely by the attenuation of the reflection from the substrate/film interface because of $x$-rays having to travel through the film. [4] A simple expression for attenuation can be written for a fixed incident and exit angle $\theta$ with respect to the surface as

$$
I_{\text {specular }}=I_{\mathrm{Pt} / \mathrm{Si}} e^{-2 \mu \rho t / \sin \theta} \cdot+I_{\mathrm{Pt}}
$$

where $t, \mu$, and $\rho$ are thickness, mass absorption coefficient and mass density, respectively. $I_{\mathrm{Pt} / \mathrm{Si}}$ is the reflected intensity from the platinum and silicon interface nithout attenuation and $I_{\mathrm{Pt}}$ is the measured specularly reflected intensity from the film surface for the thickest film. Using the known values of $\mu$ and $\rho, I_{\text {specular }}$ was calculated and shown in Fig. 6 as the dashed line. The dashed line describe well the attenuated specular reflectivity without adjustable parameters. 
The widths are shown with the right-hand-side $r$ axis in Fig. 6 . Interestingly the width, which is at about $0.0045 \hat{A}^{-1}$ and unchanged up to $1000 \AA$ thick, has increased to 0.01 $\AA^{-1}$ for the films thicker than $1000 \AA$. To guide the eye a step of solid line is also shown. For convenience of discussion we will define the growth platinum films into two regimes separated by the step of the solid line; initial stage and late stage. During the initial stage, the specular reflection is dominated by the reflection from the substrate/film interface while during the late stage it is dominated by the reflection from the film surface.

\section{A. Initial stage growth: Island regime}

As we deposited initially on a bare silicon substrate while monitoring the diffuse intensity at $Q_{x}=0.1 \AA^{-1}$, the diffuse intensity rapidly increased. After 2 minutes of deposition which corresponding to $35 \AA$ thick deposition, the intensity was an order of magnitude larger than the initial scattering from the bare silicon and the first full scan of off-specular diffuse scattering was made. In this scan the shoulders around $\pm 0.1 \AA^{-1}$. were observed. As the film thickness increases, the subsequent scans showed that the shoulders move continuously toward $Q_{x}=0$. We found that these scans were azimuthally symmetric, i.e., the scans made for several azimuthal angles' by rotating the sample about the surface normal were essentially identical. In other words, the diffuse scattering forms a halo.around the specular reflection. This is similar to the diffuse scattering previously observed for a growth of tungsten on tungsten by low-energy electron diffraction (LEED) measurements. [5] In this LEED study, the authors have attributed the ring diameter to the average distance between the islands. Their explanation of the halo of scattering is based on an assumption that the diffuse scattering is the first order. two-dimensional powder diffraction peak from a shortrangedly ordered array of islands.

In our growth condition, however, we have no reason to believe that the islands should form an ordered array. In fact, the nucleation of islands should occur randomly on the surface. The substrate is basically a flat amorphous silicon oxide and do not have any 
preference for nucleation of the islands either positionally nor orientationally. Therefore, $\pi$ will try to understand the halo of scattering around the specular reflection in the context of random islands growing on a substrate.

On a close examination of the $35 \AA$ thick film, there is also a diffuse scattering close to the specular reflection. Its presence is clear only for the thin samples whose thickness is smaller than $70 \AA$. As we will see in the fits, the halo:of scattering can be well accounted by the second term in the bracket of Eq. (8) and the scattering near the specular reflection is the result of the third term existing due to $\Lambda^{\prime} \neq 0$. Then why does Eq. (8) make sense for island growth?

We begin with a simple picture. Let us suppose that the initial nucleation of islands will occur in a completely random manner on the surface. As they grow, some of them may touch each other and coalesce together to form a-larger. island. If this process continues, the islands will randomly.occupy the entire surface area with an equal probability except that no islands can overlap each other. Therefore, the probability of finding the nearest neighbor island from an island will be zero for the distance smaller than the sum of the radii of the pair and a constant for the distance larger than the sum. This case can be represented by a pair distribution function $W(x)$ shown in Fig. 2(a).

Let us now consider a more sophisticated model of island growth: At initial stage of island growth; nucleation of islands will occur for small sizes of atomic clusters, as small as a few atoms. Once nucleation of islands occurs they will grow via surface diffusion of atoms arrived on the surface. As the sizes of islands grow, edges of some islands will grow close each other. When a pair of islands are closer than an average diffusion length, the growth between them can slow down, therefore, effectively they move apart. To illustrate this point, a nearest neighbor pair of islands are schematically shown in Fig. 7. In the figure, the twro checkered circles indicate the pair of islands. The length $L$ indicates roughly a diffusion length of the atoms arrived at the surface. The white area then indicates roughly the area. from which the islands receive the supply of atoms to their perimeters. Since the white area per unit length of the island perimeter is considerably smaller for between two islands than 
the rest of the white area, the growth at the edge will be slower betwreen the islands than the rest. The growth rates are approximately shown as the lengths of the arrows at various directions. As a result, the two islands will effectirely move apart as they. grow and the distance between them increases.

In this model, therefore, the probability of finding nearest neighbor island will increase at the average contact distance and the pair distribution function will have a peak at $x \approx 2 a$. In an ideal case, all the initial nucleation sites grow to islands without coalition. Then, the pair distribution function will have a delta-function sharp peak whose integrated area is the same as the integrated area of the dip (the total probability of hard-core exclusion). In reality the peak will be broad and we will approximate the pair distribution function for this case to Fig.2(b). Here the peak in the pair distribution is approximated by a constant excess probability $\left(\Lambda^{\prime}\right)$ extended between $2 a$ and $n a$. Since the integration of $W(x)-1$ in $x$ should be zero, the value $n$ can be found to be $2 \sqrt{1+\Lambda / \Lambda^{\prime}}$ as we discussed in Sec. IIIA and IIIB. The model discussed above is valid if the surface coverage is small and the surface diffusion is dominating mechanism for island growth. when the diffusion length is comparable to or larger than the average diameter of the islands. When the island sizes are large compared to the surface diffusion length of the arriving atoms or the coverage is high, other possibilities such as island coalition become more important. Then the value of $\Lambda^{\prime}$ becomes negligibly small and the situation becomes simpler.

In addition, since the size of islands continuously grow while new nucleations occur, a wide distribution of island sizes is expected. Without knowing the precise statistical distribution, we will assume the distribution shown in. Eq. (7). The characteristics of this distribution is that a gradual distribution from $a=0$ to the mean value but exponentially diminishing probability beyond the mean value. These characteristics of this function is quite realistic for the island growth because the largest size of the islands will be limited to the sizes of oldest islands but the smallest size can be as small as the nucleation size which are just nucleated.

The scans were fit with Eq. (8) and examples of the fits are shown in Fig. 8. The top scan 
is for the $35 \hat{A}$ thick film and the thickness of the films are progressively thicker for the scans displayed below it. The scans were offset for clarity. The data points for $\left|Q_{x}\right|>4.0 \mathfrak{A}^{-1}$ display a significant intensity drop by the edge of the $x$-rar window of the chamber and were not included in the fits. For the top few scans, the diffuse scattering immediately around the specular reflection is noticeable. As shown in Fig. 3, it results from a considerable amount of $\Lambda^{\prime} \neq 0$ contribution. As the thickness of the film increases, it decreases and the shoulder moves in closer to the origin, which indicates that the island size is increasing. In Fig. 9, two fit parameters are plotted. The filled circle indicates the average island size and the open circles indicate the exponent for the average form factor as we discussed in Sec. IIIB. The average island size monotonically increases and the exponent $(\chi+1)$ monotonically decreases as the film thickness increases. The solid lines are guides to the eye. The other fit parameters $\Lambda$ and $\Lambda^{\prime}$ were $0.8(1)$ and $0.23(9), 0.9(1)$ and $0.13(9), 1.0(1)$ and $0.08(9)_{\text {; }}$ and so forth.

The average island size is $30(3) \AA$ for the $35 \AA$ thick film. Since the lateral size and the average thickness are similar, the shape of the islands must be quite spherical. When the island shape is spherical, the exponent $1+\chi$ for the average form factor has to be 3. As we can see in Fig. 9, the value of $1+\chi$ is $\sim 3.7(2)$. As further deposition occurs: the coverage rapidly saturates to the full coverage. That means that the whole surface is uniformly covered by the islands or lumps of the platinum and the structure coarsens as the deposition continues. During this period, the shape and the distribution of the islands also rapidly changes as indicated by the rapid drop of the exponent $1+\chi$ to 1.5 . This exponent means that the islands look more like disks and the distribution is wide as in Eq. (7). This is quite reasonable.because the islands will look like spheres or hemispheres due to the relatively large surface tension and the size distribution is narrower when the size is small. However, the shape of the island will become flatter and the distribution will widen as the island size increases. 


\section{B. Late stage growth: Scaling regime}

We begin this section by deriving an equation with which we can extract the finite size scaling exponent. The diffuse scattering from a single self-affine rough surface can be written in a form similar to Eq. (2.18) of Ref. [7] as

$$
I(\mathbf{Q})=\frac{2 \pi}{Q_{z}^{2}} \int_{0}^{D} d x x e^{-Q_{z}^{2} G(x, t) / 2} J_{0}\left(Q_{11} x\right)
$$

where $G(x)$ is height-height distribution function. Since the coherency of x-rays does not end abruptly the coherence length $D$ is loosely defined quantity and represents an average value of many different coherence lengths for many $x$-rays. By approximating that $G(x)$ is. composed of two parts [1] we get

$$
I(\mathrm{Q})=\frac{2 \pi}{Q_{z}^{2}} \int_{0}^{x_{0}} d x x \eta(x) J_{0}\left(Q_{\sharp} x\right)+\frac{2 \pi}{Q_{z}^{2}} e^{-Q_{z}^{2} \sigma^{2}(t)} \int_{0}^{D} d x \dot{x} J_{0}\left(Q_{n} x\right)
$$

where the function $\eta(x)=e^{-A^{2} Q_{z}^{2} x^{2} x / 2}-e^{-Q_{x}^{2} \sigma^{2}(t)}$. The second term in Eq. (10) is the specular reflection and the first term is the diffuse scattering that we measure in our experiments. The behavior of the function $\eta(x)$ is relatively simple. It decreases monotonically from $1-e^{-Q_{x}^{2} \sigma^{2}(t)}$ at $x=0$ to 0 at $x=x_{0}$. By using a integral by part, we can see that the first. term can be rewritten as

$$
I_{\text {diffuse }}(\mathrm{Q}) \approx \frac{1}{Q_{\mathrm{ll}}} \int_{0}^{x_{0}} d x x^{2 \chi} e^{-A^{2} Q_{x}^{2} x^{2 x} / 2} J_{1}\left(Q_{11} x\right)
$$

In general, the evaluation of the integral in Eq: (11) does not have a closed form and we will not attempt to.numerically or analytically evaluate it. Instead, we will approximate it to a mathematically sound functional form. which has at least accurate limiting behaviors. Now let us focus on the asymptotic behavior of $I_{\text {diffuse }}(Q)$. For large value of $Q_{\| 1}$ the integral is dominated by $x \approx 0$ behavior and $x_{0}$ can be replaced by $\infty$. Then $I_{\text {diffuse }}(\mathrm{Q}) \propto Q_{11}^{-2-2 \chi}$. [8] For small value of $Q_{11}$, the integration becomes constant $\left(Q_{11}\right.$ independent) because $J_{1}(y \sim$ 0) $\propto y$. Therefore, we will choose a function

$$
I_{\text {diffuse }}(\mathrm{Q}) \propto 1 /\left[1+Q_{\mathrm{n}} \epsilon\right]^{2+2 \chi}
$$


satisfying these two limiting cases. Here $\epsilon, \chi$, and the overall scale factor are the adjust parameters in our data analyses. Incidently this form is similar to Eq. (8) when $\Lambda, \Lambda^{\prime}=0$. The finite size scaling exponent $\chi$ here is unrelated to the exponent $\chi$ in Sec. III B which is the result of averaging the form factors of the random hard core objects. We will call $\epsilon$ finite size parameter analogous to the parameter $a_{0}$ although they are unrelated. The parameter $\epsilon$ acts as the smallest unit of the surface so that Eq. (11) can be evaluated in an asymptotic r. limit for $Q_{\| 1} \gtrsim 2 \pi / \epsilon$. We can view it as the cutoff for the smallest length scale with which the surface is self-affine. The value of $\chi$ that we find here is somewhat larger than previously. observed for gold films using scanning tunneling microscopy (STM) measurements but well within current theoretical-predictions for $2+1$ dimensions.

Let us now turn the attention back to Fig. 6. Earlier we remarked that the width of . the specular peak increases at around $1000 \AA$ thickness. The thickness at which the width increase rapidly nearly coincides with the thickness at which the specular reflectivity from the surface is nearly: totally attenuated. From this observation we can conclude that the width of the specular reflection from the substrate/film interface is considerably narrower than that from the film surface. This can be.attributed to the fact that the coherence lengths of $x$-ray photons are indeed finite and its effect has to be considered in general. If the $x$-ray . coherence length was infinite, the specular width should have been resolution-limited in both - cases. One can argue that the peak is broad it is not specular because the self-affine surfaces do not have true specular reflection. This is true only if the surface is self-affine in all length scales. In reality; it is very difficult to realize a true self-affine surface and self-affine nature is generally limited to finite length scales: Therefore, the surface should look like a Gaussian random surface and the specular reflectivity should be resolution-limited if $x$-ray coherence length is indeed infinite [i.e. if $D$ in Eq. (9) is infinite]. The surface orientation averaged over the finite x-ray coherence length $D$ fluctuates as individual photons scatter from different parts of the sample. Another important implication of this observation is that reflections from the substrate/film interface and from the film surface interfere only if they are parallel each other. In other words, the specular reflection from the substrate interfere selectively 
with the reflection from the part of the surface whose average orientation is parallel to the substrate orientation. In essence, an incoherent average of the reflectivity is essential in order to extract the information of interest from the specular reflectivity measurements. [1]

The non-specular component comes only from the film surface and can be understood by the off-specular scattering from a single partially self-affine surface. One of the important characteristics of dynamic scaling of growing interface is that the finite size scaling exponent $\chi$ does not change in time or in film thickness. [9] The main focus of this section is to show that the exponent $\chi$ can be found in our off-specular diffuse scattering measurements using Eq. (12) and it does not significantly evolve during sputter deposition of the film. The dynamic scaling behavior of the sputter-deposited films during growth has been established for gold films [1] and for platinum films. [6] grown on polished silicon substrates from series of specular reflectivity scans. In the previous gold film study [1] the finite size scaling exponent $\chi$ was estimated from a STM measurements in the absence of off-specular reflectivity data. In this study the off-specular reflectivity scans are corrected for Lorenz-polarization factor and fit with Eq. (12). The fit parameters, finite size and the exponent, are plotted in Fig. 10. The exponent $\chi$ is approximately: 0.5 and basically unchanged within the size of error bars beyond $1000 \AA$ thickness.

\section{CONCLUSION}

In this proceeding, we measured a series of off-specular x-ray reflectivity scans during platinum deposition. It is shown that islands grow on silicon substrates at early stage of growth evidenced from halo of off-specular scattering around the specular reflection. The offspecular was analyzed based on a random statistical model of islands grown on the surface. It is also shown that the film surface grows consistently with the dynamic scaling behavior, that is, the finite size scaling exponent $\chi$ is constant over large period and its value is in reasonable agreement with the theoretical predictions. A more accurate and complete study of the scaling behavior is planned. 
This work was performed under DOE contract No. W-31-109-ENG-38. 


\section{REFERENCES}

[1] H. You, R. P. Chiarello, H. K. Kim, and K. G. Tanċ=toort, Phys. Rev. Lett. 70, 2900 (1993).

[2] J. Q. Zheng, M. C. Shih, X. K. Wang, S. Williams. P. Dutta, R. P. Chang, and J. B. Ketterson, J. Vac. Sci. Technol. A 9, 128 (1991:-

[3] P. Debye, J. Math and Phys. 4, 153 (1925)

[4] There will be a contribution to the specular intensity irom the film surface. However, the film surface is considerably rougher than the film/substrate interface and its contribution to the total intensity is a small fraction until the reflection from the film/substrate interface become sufficiently attenuated.

[5] P. Hahn, J. Clabes, and M. Henzler, J. Appl. Phys. 51, 2079 (1980)

[6] Unpublished.

[7] S. K. Sinha, E: B. Sirota, S. Garoff, and H. B. Stanlet. Phys. Rev. B, 382297 (1988).

[8] This asymptotic behavior can easily be shown numerically as well as analytically for $\chi \sim 0.5$.

[9] J. M. Kim and J. M. Kosterlitz; Phys. Rev. Lett. 62. 2289-2292 (1989); J. M. Kim, J. M. Kosterlitz, and.T. Ala-Nissila, J. Phys. A: Math Gen. 24, 5569 (1991): 


\section{FIGURES}

FIG. 1. (a) A schematic drawing of the sputtering/x-ray chamber. (b) A close-up view of target assembly.

FIG. 2. (a) and (b) are possible pair distribution functions discussed in the text. The difference (c) from (d) is equivalent to (b) and they were used in Sec. IIIA.

FIG. 3. A plot of Eq. (8) for several $\Lambda$ and $\Lambda^{\prime}$ possible values in a semi-log scale.

FIG. 4. A series of off-specular x-ray reflectivity data measured during platinum film growth.

FIG. 5. The last off-specular scan of the series plotted in linear and in semi-log scale. The film was approximately $9000 \AA$ thick.

FIG. 6. The specular'peak intensity and its width vs. film thickness plotted in a log-log scale.

FIG. 7. Growth of two nearest neighbor islands are schematically shown. See the text for details.

FIG. 8. Examples for the fit of the data to the random island model discussed in the text are shown.

FIG. 9. The fit parameters, average islands size and the exponent associated with the form factor, vs. the film thickness.

FIG. 10. The exponent $\chi$ and the finite size parameter are shown. See the text for discussion. 

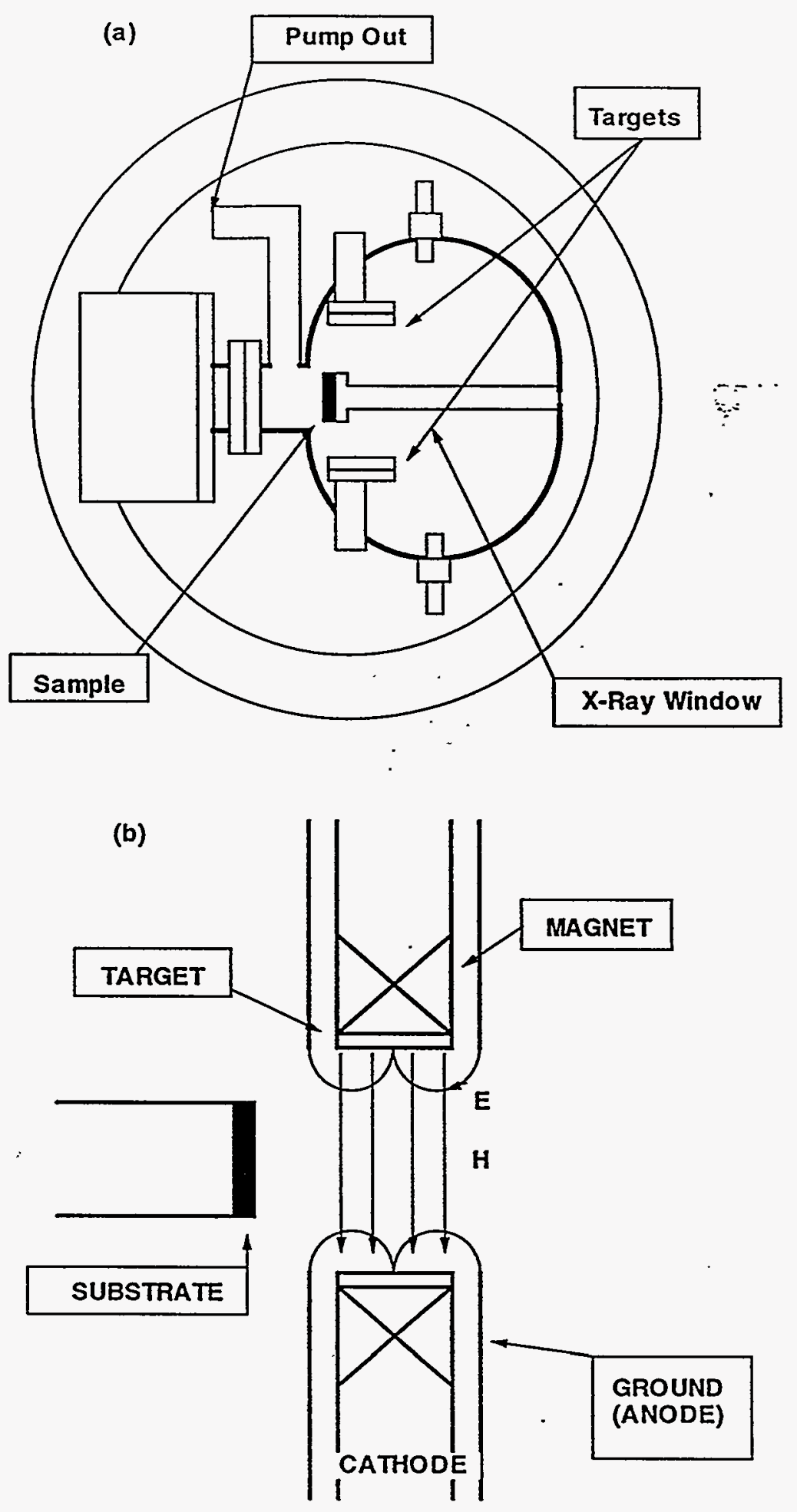

FIG. 1 


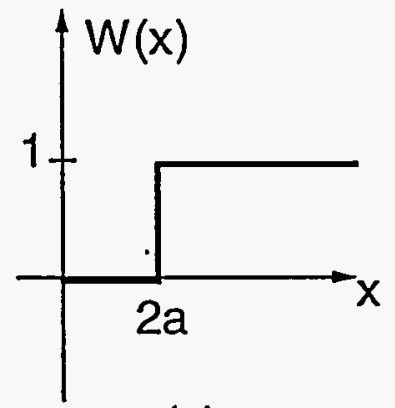

(a)

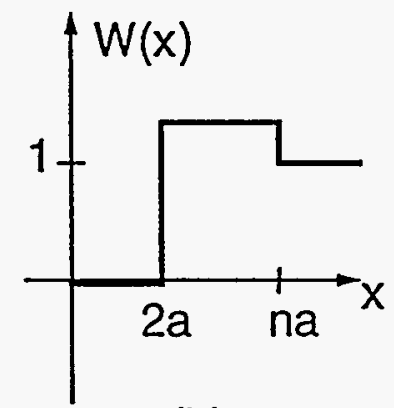

(b)

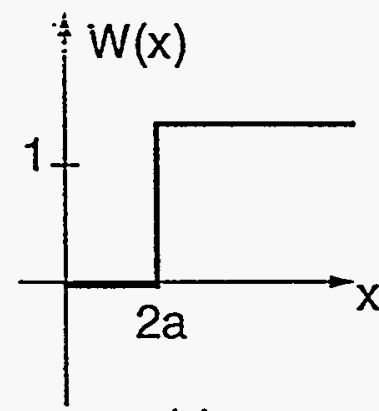

(c)

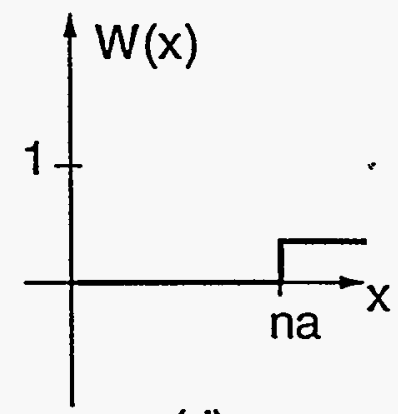

(d)

FIG. 2 


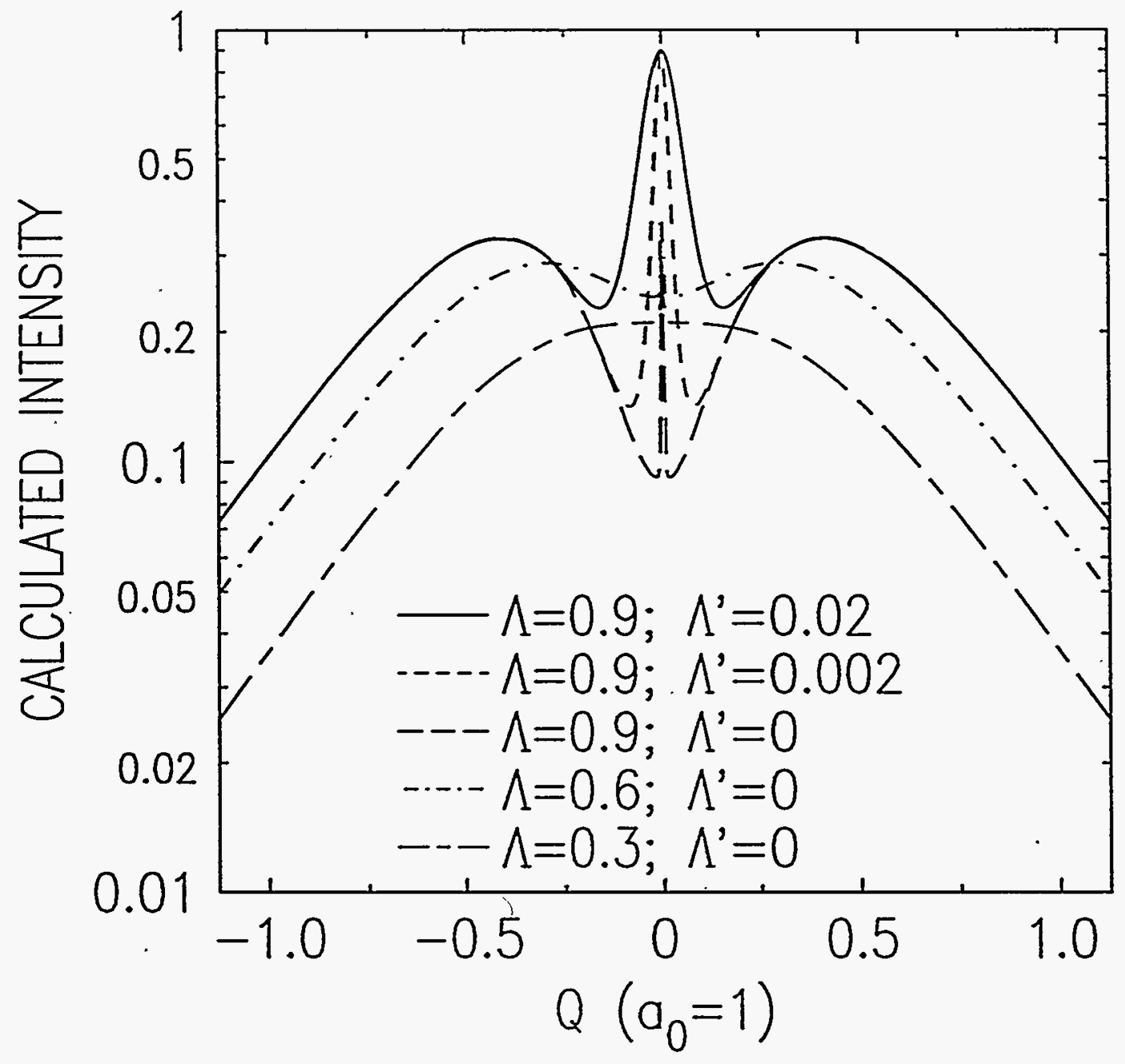

FIG. 3 


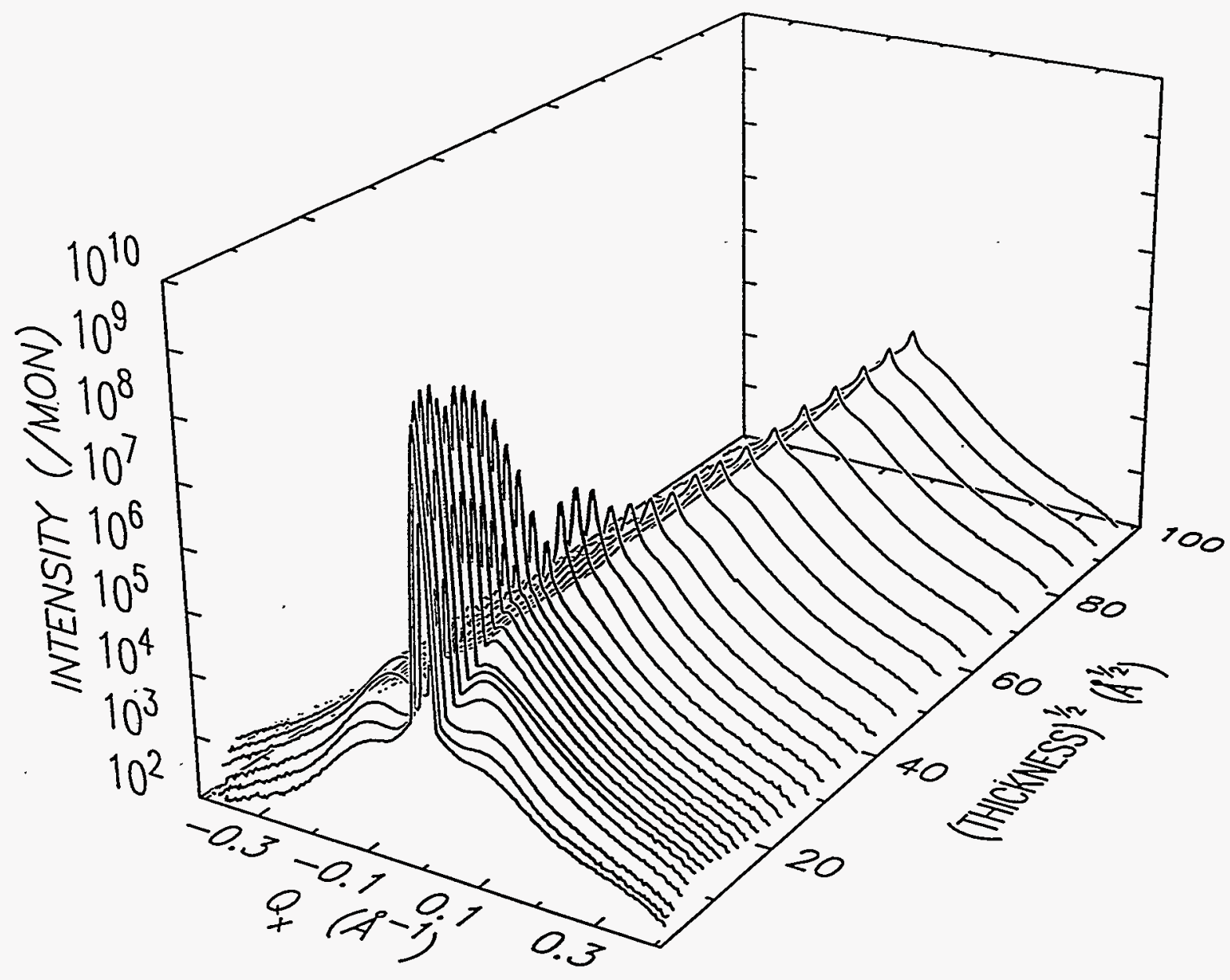

FIG. 4 


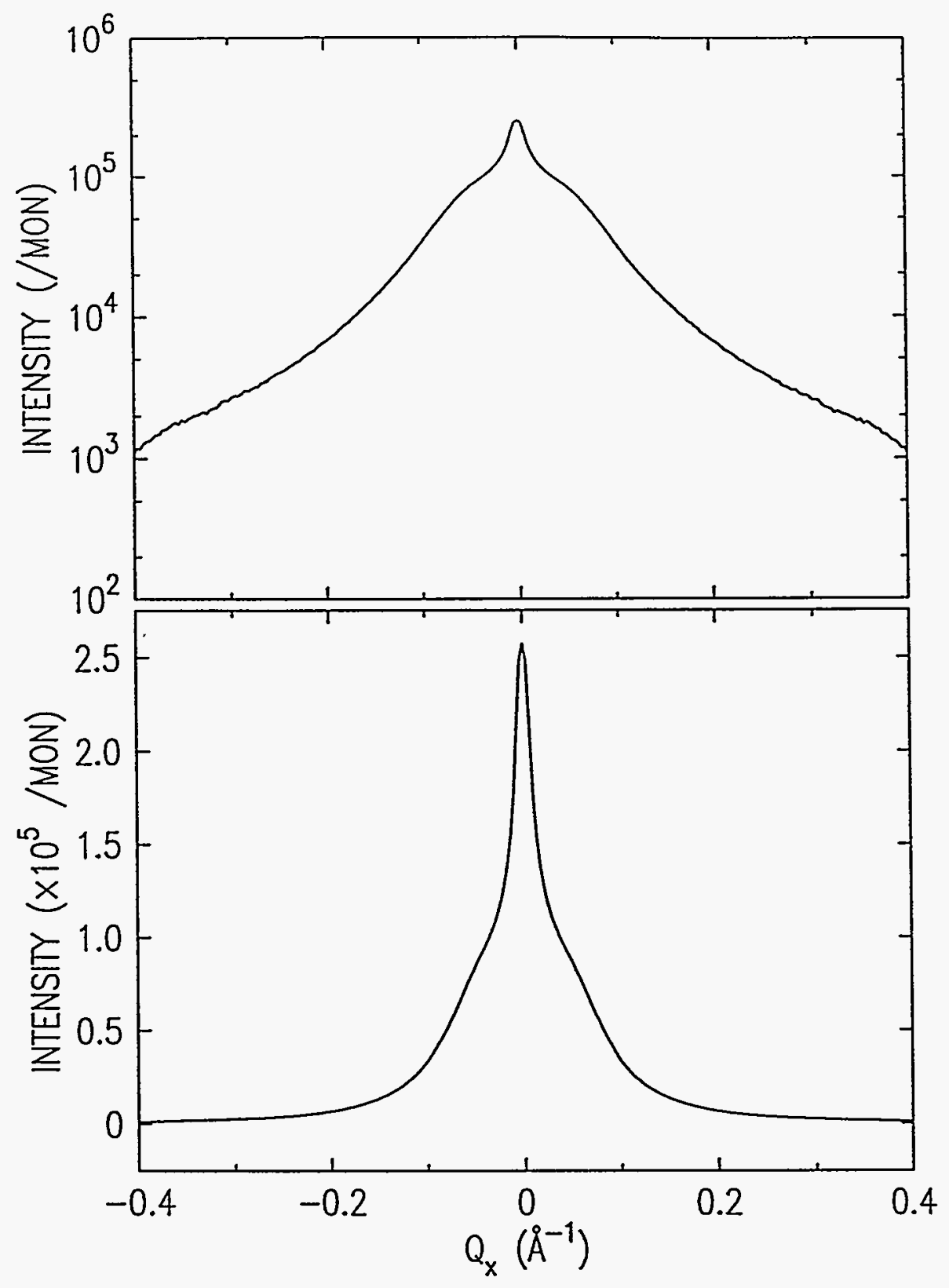

FIG. 5 


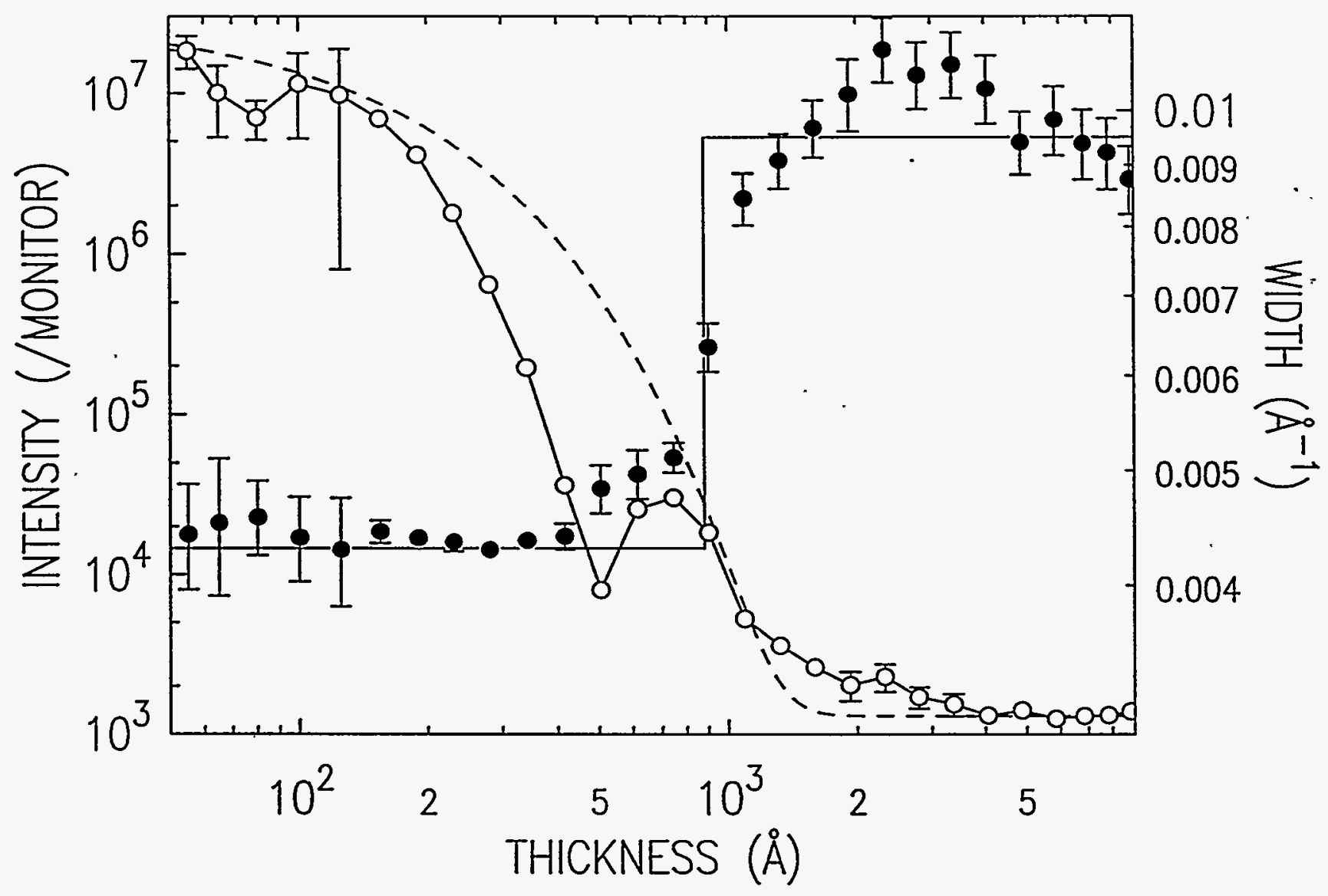

FIG. 6 


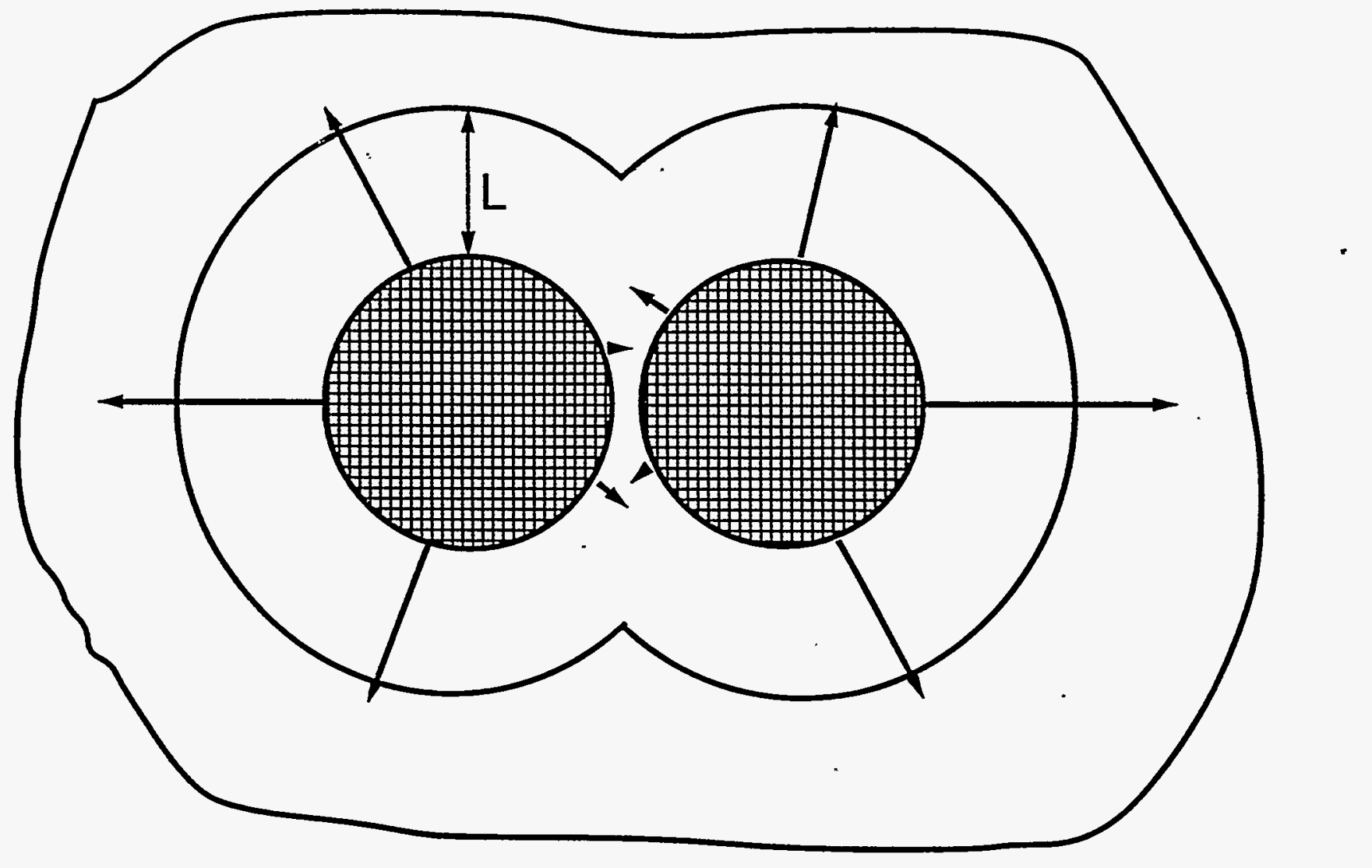

FIG. 7 


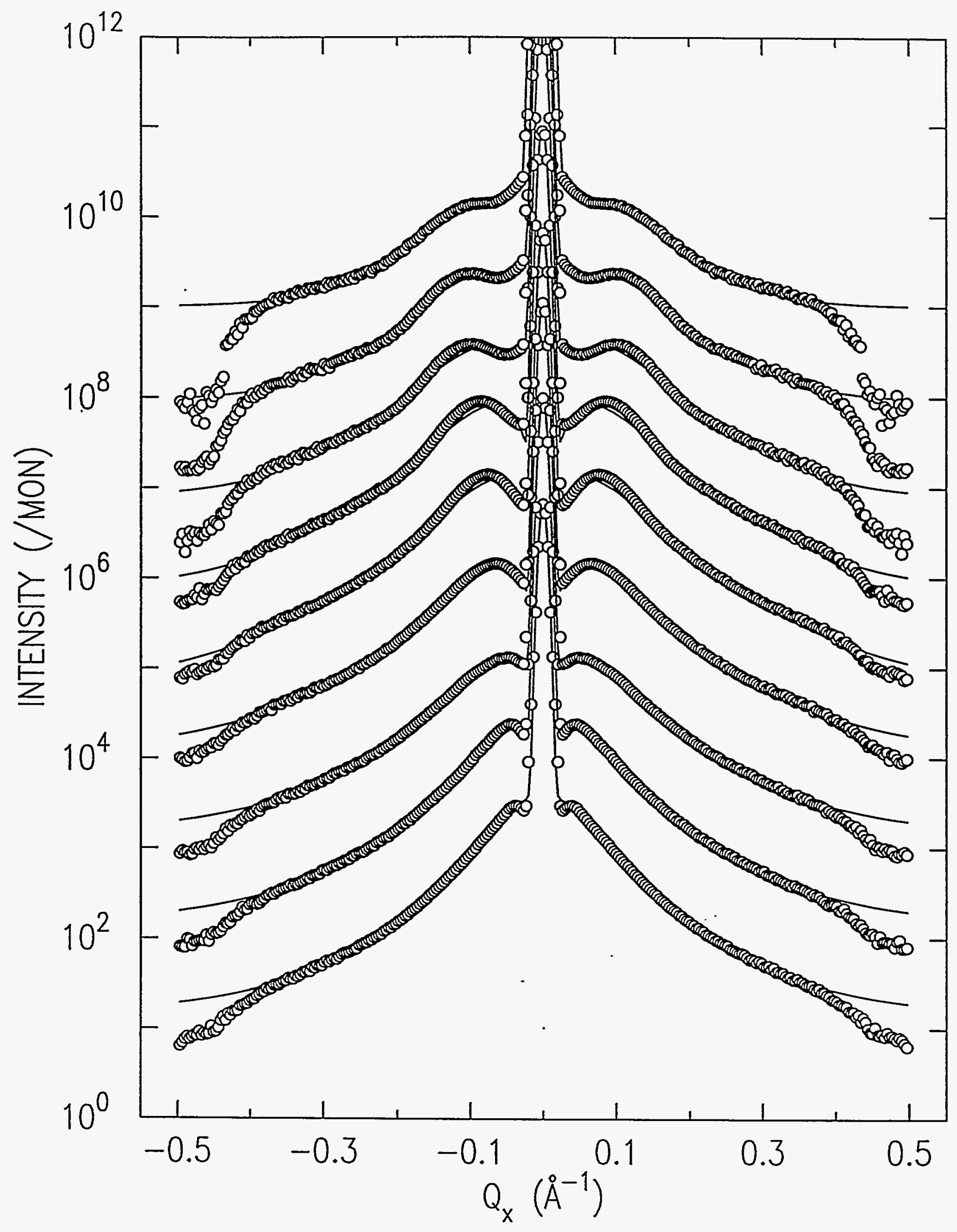

FIG. 8 


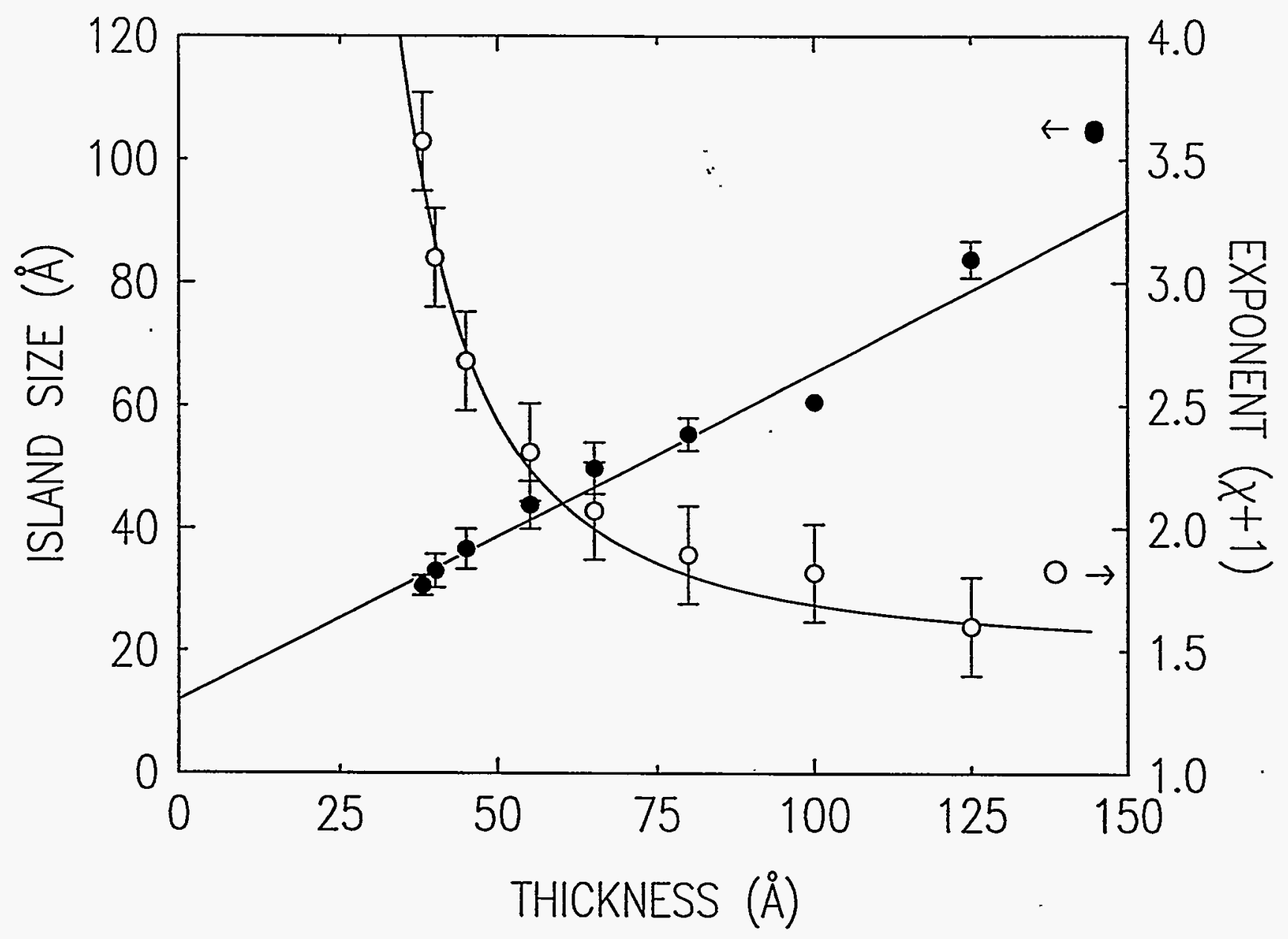

FIG. 9 


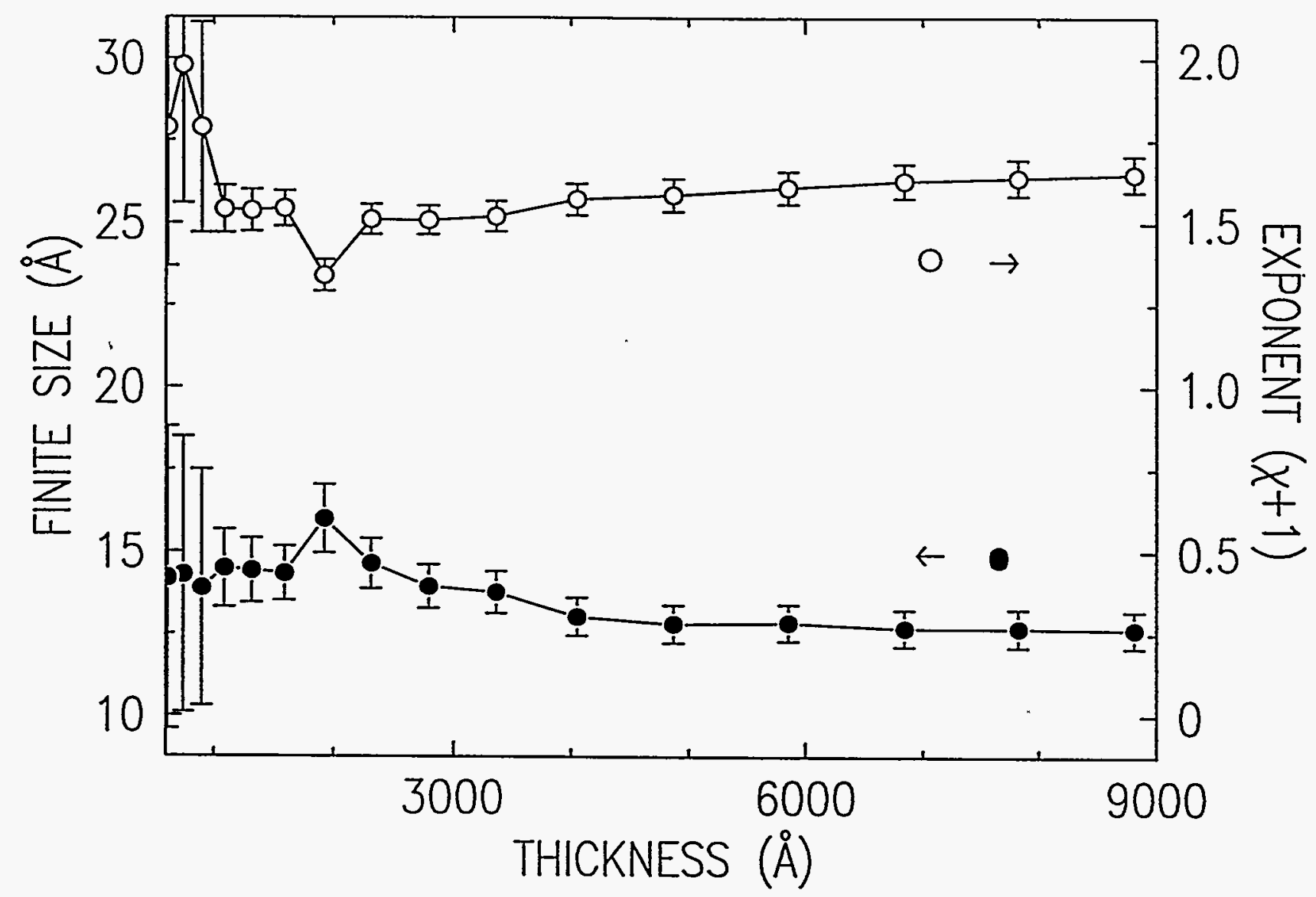

FIG. 10 